\title{
Thyroid hormones profile of patients with type 2 diabetes mellitus in Kano, Nigeria
}

\author{
Zainab Abubakar Mustapha, Kabiru Abdulsalam, Isah A. Yahaya \\ Department of Chemical Pathology and Immunology, Bayero University/Aminu Kano Teaching Hospital, \\ Kano, Nigeria
}

\begin{abstract}
Diabetes mellitus (DM) and thyroid disease are the two most common endocrine disorders in the general population. Several Studies have shown that thyroid dysfunction is common in patients with DM, and thyroid dysfunction have been found to have a considerable impact on the glycaemic control and often increases the risk of development of long-term complications in patients with diabetes mellitus. This study determined the prevalence of thyroid dysfunction in patients with type $2 \mathrm{DM}$ in Kano, North-Western Nigeria. The study was a descriptive cross-sectional study conducted on 250 participants made up of 130 patients with type $2 \mathrm{DM}$ and 120 apparently healthy non-diabetic controls. Questionnaires were used to collect information on bio data, medical history, duration of diagnosis of diabetes and type of treatment. Also, blood samples of the participants were collected and analyzed for fasting plasma glucose, $\mathrm{fT}_{3}, \mathrm{fT}_{4}$, and $\mathrm{TSH}$. The results were interpreted using American Thyroid Associations' criteria and the data was analyzed using the statistical software package, STATA version 20.
\end{abstract}

Correspondence: Kabiru Abdulsalam, Department of Chemical Pathology and Immunology, Bayero University/Aminu Kano Teaching Hospital, Kano, Nigeria.

Tel.: +2348039749586

E-mail: kabdulsalam.cpat@buk.edu.ng

Key words: Diabetes Mellitus, free Tri-iodothyronine, free Thyroxine, Thyroid Stimulating Hormone.

Contributions: The authors contributed equally in the study.

Conflict of interest: The authors declare no conflict of interest.

Ethics approval and consent to participate: Ethical approval was obtained from the Health Research Ethics Committee (HREC) of the Hospital. Guideline and Provisions from the Helsinki declaration were properly followed and respected at every stage of the study.

Availability of data and materials: All data and materials are available within the text.

Received for publication: 9 June 2020

Accepted for publication: 9 July 2020.

This work is licensed under a Creative Commons Attribution NonCommercial 4.0 License (CC BY-NC 4.0).

(C) Copyright: the Author(s),2020

Licensee PAGEPress, Italy

Annals of African Medical Research 2020; 3:112

doi:10.4081/aamr.2020.112
Two hundred and thirty-four (93.6\%) of the participants were euthyroid while sixteen $(6.4 \%)$ were found to have various forms of thyroid dysfunction. The prevalence of thyroid dysfunction was $10 \%$ and $2.5 \%$ among type 2 diabetics and controls respectively. Among the type $2 \mathrm{DM}$ patients with thyroid dysfunction, $38.5 \%$ had hypothyroidism. Thyroid dysfunction was found to be commoner among type $2 \mathrm{DM}$ patients than non-diabetic individuals with hypothyroidism being the commonest disorder.

\section{Introduction}

Diabetes Mellitus (DM) and Thyroid disease are two most common endocrinopathies seen in the general population. ${ }^{1} \mathrm{DM}$ refers to a group of metabolic disorders characterized by chronic hyperglycaemia due to insulin deficiency (absolute or relative), insulin resistance or both. ${ }^{1,2}$ Globally, an estimated 422 million adults were living with diabetes in 2014, compared to 108 million in $1980 . .^{3}$ The global prevalence of diabetes has nearly doubled since 1980 , increasing from $4.7 \%$ to $8.5 \%$ in the adult population. ${ }^{3}$ This reflects an increase in associated risk factors such as being overweight or obese.

In the year 2015, the International Diabetes Federation (IDF) estimated that 415 million individuals have diabetes in the world and more than 14 million people are found in the African continent, and that by 2040 , this figure may likely double. ${ }^{4}$ In Nigeria, there were more than 1.56 million diabetics in 2015 among adults between the ages of 20-79 years with an estimated prevalence of $1.9 \%{ }^{4}$

Thyroid disease on the other hand is equally common in the general population with a higher prevalence in diabetics than in non-diabetic individuals. ${ }^{5}$ In a study conducted in Lagos, Nigeria, the prevalence of thyroid dysfunction in type 2 diabetic patients was $8.3 \%{ }^{6}$ Similarly, a study conducted in Ibadan, Nigeria, reported the prevalence of thyroid disease to be $29.7 \%$ in type 2 diabetics compared with $2.8 \%$ in non-diabetic individuals. ${ }^{7}$ This signifies higher prevalence of thyroid disorder in type 2 diabetics than in non-diabetics.

Insulin and thyroid hormone are both intimately involved in cellular metabolism. They influence several aspects of carbohydrates, proteins and lipids metabolism. ${ }^{8}$ Therefore, alteration in the levels of the either of the two hormones may result in functional derangement of the other. Previous studies have shown that thyroid hormones are insulin antagonist with high levels being diabetogenic, while reduced levels of the hormone are associated with lower chances of developing DM. ${ }^{9-11}$

The co-existence of diabetes and thyroid disorders has been associated with increased morbidity and mortality. ${ }^{12}$ Presence of thyroid dysfunction may have profound influence on blood glucose control and may exacerbate chronic complications of type 2 DM. This has been amply demonstrated by several studies. ${ }^{7,13}$

Though both hyperthyroidism and hypothyroidism may affect 
the course of diabetes mellitus, their effects are different. Hyperthyroidism is typically associated with worsening of glycaemic control and increased insulin requirement. ${ }^{5}$ This is mainly because it causes increased hepatic gluconeogenesis, gastrointestinal glucose absorption and probably insulin resistance. This partly explains why glycaemic control deteriorates when diabetic patients develop hyperthyroidism. ${ }^{10}$ Hyperthyroidism may occasionally unmask patients with latent diabetes. ${ }^{9}$ However, in hypothyroidism the reduced rate of insulin degradation may lower the exogenous insulin requirements, but it rarely causes significant changes in glycaemic control. ${ }^{5}$

Hyperthyroidism increases the risk of heart disease in diabetics by increasing heart rate and consequent development of cardiac arrhythmias. It may also lead to angina, worsen cardiac failure or make its treatment somewhat difficult. The combination of hyperthyroidism and diabetes mellitus also increases the risk of pathological fractures and disability, especially in the elderly. ${ }^{9}$

Hypothyroidism is accompanied by several abnormalities in plasma lipid metabolism such as elevated triglyceride and lowdensity lipoprotein cholesterol concentrations. ${ }^{5}$ Even subclinical hypothyroidism may exacerbate the co-existing dyslipidaemia commonly encountered in type 2 diabetes and further increases the risk of cardiovascular diseases. ${ }^{5}$ Dyslipidaemia was found to be present in all hypothyroid type 2 diabetics as compared to $35.5 \%$ of their euthyroid counterparts, ${ }^{7}$ and therefore, adequate thyroxine replacement may likely reverse the lipid abnormalities. ${ }^{5}$ Previous studies have also shown increased prevalence of other diabetic complications such as nephropathy, cerebro-vascular events and sight threatening form of retinopathy among type 2 diabetic patients with subclinical hypothyroidism. ${ }^{10}$

In the same vein, various treatment modalities currently used to manage patients with DM have also been shown to affect thyroid hormone levels. Several studies have shown that insulin treatment or insulin resistance with resultant high plasma insulin levels in type 2 diabetes may predispose patients to deranged thyroid function. ${ }^{7-9}$ This is because insulin, an anabolic hormone, increases the levels of $\mathrm{fT}_{4}$ by inhibiting the hepatic deiodination of $\mathrm{T}_{4}$ to $\mathrm{T}_{3}$. 1,9

The use of oral hypoglycaemic medications in the treatment of patients with type 2 diabetes also has varying effect on thyroid hormone homeostasis. A pilot study of type 2 diabetic, hypothyroid patients on metformin revealed a baseline reduction in thyroid stimulating hormone (TSH) levels after 6 months of treatment. $1,10,11$

This study was therefore aimed at determining the prevalence of thyroid dysfunction in patients with type 2 diabetes mellitus in Kano, North-Western Nigeria.

\section{Materials and Methods}

Systematic sampling technique was used to carefully recruit the study participants from both endocrinology and metabolic clinics of Aminu Kano Teaching Hospital, (AKTH), while the blood samples were analyzed in the Hospital's Chemical Pathology Department.

The study was conducted on type 2 diabetic patients and apparently healthy non-diabetic individuals who were patient relatives, voluntary blood donors and hospital staff served as controls.

Excluded from the study were patients with significant comorbidities, autoimmune diseases, history of thyroid dysfunction prior to diagnosis of DM, past history of irradiation or surgery to the neck, pregnant and lactating women, and those with acute ill- nesses. Ethical approval was obtained from the Health Research Ethics Committee (HREC) of the Hospital. Guideline and Provisions from the Helsinki declaration were properly followed and respected at every stage of the study.

Participants were counseled on the protocols of the study and blood samples were collected between 8-9 am for fasting plasma glucose and thyroid function profile ( $\mathrm{fT}_{3}, \mathrm{fT}_{4}$ and $\left.\mathrm{TSH}\right)$.

Quantitative measurements of serum TSH, fT3and fT4 were determined using a highly sensitive and specific electrochemiluminescent (ECL) technology on elecsys 2010 automated immunology analyzer (Roche- Hitachi). ${ }^{14-16}$ Glucose oxidase method was used for plasma glucose measurement. ${ }^{17}$

All data generated were entered into excel spreadsheets, cleaned and then exported into STATA statistical package version 13 (StataCorp., College Station) for statistical analyses. ${ }^{18}$

A confidence interval of $95 \%$ was used and the level of statistical significance was taken as a $\mathrm{P}$ value of $<0.05$.

\section{Outcome measures}

The following reference intervals were used for the interpretation of results. TSH $0.3 \mathrm{mIU} / \mathrm{L}-4.2 \mathrm{mIU} / \mathrm{L} . \mathrm{fT}_{3}=3.1 \mathrm{pmol} / \mathrm{L}-$ $6.8 \mathrm{pmol} / \mathrm{L} . \mathrm{fT}_{4}=10.0 \mathrm{pmol} / \mathrm{L}-22.0 \mathrm{pmol} / \mathrm{L}$

\section{Results}

The socio-demographic characteristic of the study participants is shown in Table 1 .

The mean age of all the subjects was $50.9 \pm 14.5$ years, while the corresponding mean age for the subjects and controls were 51.7 \pm 12.2 years and $50.2 \pm 11.7$ years respectively.

There was female preponderance among the subjects, with male to female ratio of approximately $1: 2$ and $1: 1$ for the subjects and controls respectively.

Majority of the subjects in both groups studied were married and the most of them were unemployed.

Table 2 shows the thyroid function profile of the study participants.

There was a statistically significant difference in the mean serum $\mathrm{fT}_{3}$ and $\mathrm{fT}_{4}$ between the patients and the controls $(\mathrm{P}=$ 0.0088).

The prevalence of thyroid dysfunction was $10 \%$ and $2.5 \%$

Table 1. Socio-demographic characteristics of study participants.

\begin{tabular}{lcc} 
Socio-Demographic Variables & Patients (\%) & Controls (\%) \\
Mean age (years) & $51.7 \pm 12.2$ & $50.2 \pm 11.7$ \\
Sex & & \\
$\quad$ Male & $43(33.1)$ & $59(49.2)$ \\
Female & $87(66.9)$ & $61(50.8)$ \\
$\quad$ Total & $130(100)$ & $120(100)$ \\
Marital status & & \\
Married & $104(80.0)$ & $79(65.8)$ \\
Single & $3(2.3)$ & $37(30.8)$ \\
Divorced & $4(3.1)$ & $2(1.7)$ \\
Widowed & $19(14.6)$ & $2(1.7)$ \\
Total & $130(100)$ & $120(100)$ \\
\hline Occupation & & \\
Civil servant & $12(9.2)$ & $50(41.7)$ \\
Business & $35(26.9)$ & $15(12.5)$ \\
Others & $83(63.9)$ & $55(45.8)$ \\
Total & $130(100)$ & $120(100)$ \\
\hline
\end{tabular}

Age is presented as mean \pm standard deviation while sex, marital status and occup. 
among participants with type 2 diabetes and controls respectively The most frequent type of thyroid dysfunction among type $2 \mathrm{DM}$ subjects was hypothyroidism (Table 3 ).

\section{Discussion}

This study recruited 250 participants, made up of 130 patients with type $2 \mathrm{DM}$, and 120 apparently healthy non-diabetic individuals as controls.

The mean age of the two groups studied were essentially similar. This was in agreement with studies by Ghazali and co-workers in Ibadan and Udiong and colleagues in Calabar. ${ }^{7,9}$ This observation was desirable because it eliminates the effect of age on thyroid function and thus the results of the two groups were comparable.

The female preponderance among the studied type 2 diabetics suggested an increasing prevalence of this non-communicable disease in females. This observation may be explained by the fact that majority of modifiable risk factors associated with the development of type $2 \mathrm{DM}$ such as physical inactivity, sedentary life style and obesity are commoner in females than in males. ${ }^{3}$

The high rate of unemployment observed among the study subjects may be due to rapid population growth, unstable and corrupt political environment, economic recession, lack of infrastructure and poor-quality education. ${ }^{18,19}$

A number of reports worldwide have shown a higher prevalence of thyroid disorders in type 2 diabetic patients compared to non-diabetics. ${ }^{5,9,12}$ The prevalence of thyroid dysfunction in this study was $10.0 \%$ in type 2 diabetics and $2.5 \%$ in non-diabetics. Similar finding were reported in other climes. A study by Smithson et al. in the United Kingdom reported a prevalence of $10.8 \%$ of thyroid dysfunction among diabetic patients registered in general practice ${ }^{5,8}$ while a prevalence of $12.3 \%$ was reported among Greek diabetic patients. ${ }^{20}$ In Jordan, Radaideh and co-workers reported that thyroid dysfunction was present in $12.5 \%$ of type 2 diabetic patients $^{21}$ and Akbar et al. observed that thyroid dysfunction was present among $16 \%$ of type $2 \mathrm{DM}$ patients in Saudi Arabia. ${ }^{22}$ Perros and colleagues also demonstrated an overall prevalence of $13.4 \%$ of thyroid diseases in diabetics with the highest prevalence in type 1 female diabetics (31.4\%) and lowest prevalence in type 2 male diabetics $(6.9 \%){ }^{23}$ Similarly, in a study conducted by Cardoso et al. in Lagos, the prevalence of thyroid dysfunction among patients with type $2 \mathrm{DM}$ was $8.3 \%{ }^{6}$

Table 2. Serum $\mathrm{fT}_{3}, \mathrm{fT}_{4}$, TSH and FPG (mean \pm SEM) of study participants.

\begin{tabular}{lccc} 
Parameter & Patients & Controls & P value \\
$\mathrm{fT}_{3}(\mathrm{pmol} / \mathrm{L})$ & $4.89 \pm 0.07$ & $5.15 \pm 0.07$ & 0.0088 \\
$\mathrm{fT}_{4}(\mathrm{pmol} / \mathrm{L})$ & $13.48 \pm 0.17$ & $14.12 \pm 0.17$ & 0.0088 \\
$\mathrm{TSH}(\mu \mathrm{I} / \mathrm{LL})$ & $1.76 \pm 0.42$ & $1.55 \pm 0.08$ & 0.6405 \\
$\mathrm{FPG}$ & $10.84 \pm 0.51$ & $4.71 \pm 0.06$ & 0.0000 \\
\hline
\end{tabular}

Table 3. Prevalence and Patterns of thyroid dysfunction among study groups.

\begin{tabular}{lccc} 
Category & Patients, N (\%) & Controls, N (\%) & Total, N (\%) \\
Euthyroid & $117(90.0)$ & $117(97.5)$ & $234(93.6)$ \\
Hypothyroidism & $5(3.8)$ & $2(1.7)$ & $7(2.8)$ \\
\hline Hyperthyroidism & $4(3.1)$ & $1(0.8)$ & $5(2.0)$ \\
\hline
\end{tabular}

Other studies in Nigeria reported much higher prevalence of thyroid dysfunction among type 2 diabetic patients compared to the prevalence observed in this study. Noted examples are studies by Ghazali et al. in Ibadan that reported a prevalence of $29.7 \%$ and another study by Udiong et al. in Calabar with a prevalence of $46.5 \%{ }^{7,9}$

The observed differences in the prevalence of thyroid dysfunction among type 2 DM subjects seen in this study and that of other studies indicated above may be due to dietary, geographical, racial and patient related factors as well as factors related to the analytical performance of the methods used. A highly sensitive and specific analytical method (electrochemiluminescent, ECL, technology on elecsys 2010 automated immunoanalyser) was used to measure all the parameters in this study. While manual technique based on ELISA principle was used in the other quoted studies. Modern automated immunology analysers have better analytical performance compared to manual methods. ${ }^{24-26}$

Hypothyroidism was the most frequent type of thyroid dysfunction observed in this study accounting for 3.8\% of type 2 diabetic subjects. This was in agreement with studies by Ghazali and co-workers and that of Udiong et al. who also reported hypothyroidism as the most prevalent thyroid dysfunction among type 2 diabetics. $^{7,9}$ The findings of low serum thyroid hormones in this study are similar to what was reported by Chen and Singh et al., who also showed significantly lower levels of free and total thyroid hormones in type 2 diabetics when compared with non-diabetic controls. ${ }^{27,28}$ Similarly, a study conducted by Swamy and coworkers concluded that type 2 diabetics had lower serum $\mathrm{T}_{3}$ and $\mathrm{T}_{4}$ levels when compared with their non-diabetic counteparts. ${ }^{29}$ These findings may be explained by the effect of DM on the functions of the hypothalamo-pituitary-thyroid axis. Hypothalamic TRH, pituitary TSH secretion rates are decreased and despite normal peripheral TSH metabolism, iodide uptake by the thyroid gland and thyroid hormones production are also diminished..$^{9,27}$ There are also important structural changes in both thyroid and pituitary glands which are accompanied by marked alterations in their secretory activities. ${ }^{13,30}$ Altered thyroid hormone levels have also been described in patients with diabetes especially those with poor glycaemic control by Gursoy and co-workers. They found that the nocturnal TSH peak was blunted or abolished, the TSH response to TRH was impaired and reduced $\mathrm{T}_{3}$ level was also observed. This low $T_{3}$ state may also be due to impairment in peripheral conversion of $\mathrm{T}_{4}$ to $\mathrm{T}_{3}$, which normalizes with improvement in glycaemic control. ${ }^{20,31}$

\section{Conclusions}

This study established that thyroid dysfunction is more prevalent among type 2 diabetics compared to their non-diabetic counterparts and that hypothyroidism is the commonest thyroid function abnormality.

\section{Recommendations}

Further to the findings of this study, all patients with T2DM should be screened for thyroid dysfunction because of the increased prevalence of this disorder in them. This is in line with the guidelines of the American Thyroid Association which recommends frequent testing for thyroid dysfunction in high risk persons including diabetic patients. ${ }^{16}$

Also, patients identified with overt or clinical thyroid dysfunction should be commenced on treatment immediately. This is 
because presence of thyroid dysfunction in diabetic patients affects blood glucose control with the attendant increase in the risk of developing diabetic complications.

\section{References}

1. Nicholas A. Type 2 Diabetes Mellitus and Thyroid Dysfunction. Afr J Diabetes Med 2015;22:21-3.

2. Kumar P, Clark M (Editors). Clinical Medicine. 7th edition. Spain: Saunders Elsevier; c2009. p. 982-93, 1029-59.

3. World Health Organization. Global Reports on Diabetes 2016: Geneva, Switzerland: WHO; 2016.

4. International Diabetes Federation. International Diabetes Federation Africa Region. www.idf.org/membership/afr/nige ria.

5. Wu P. Thyroid Disease and Diabetes. Clin Diabetes 2000;18:14.

6. Cardoso C, Ohwovoriole AE, Kuku SF. A Study of Thyroid Function and Prevalence of Thyroid Autoantibodies in an African Diabetic Population. J Diabetes Complications 1995;9:37-41.

7. Ghazali SM, Abbiyesuku FM. Thyroid Dysfunction in Type 2 Diabetes seen at University College Hospital, Ibadan, Nigeria. Nigerian J Physiolog Sci 2010;25:173-9.

8. Radaideh AR, El-Khateeb M, Bateiha AM, et al. Thyroid Function and Thyroid Autoimmunity in Patients with Type 1 Diabetes Mellitus. Saudi Med J 2003;24:352-5.

9. Udiong CEJ, Udoh AE, Etukudoh ME. Evaluation of Thyroid Function in Diabetes Mellitus in Calabar, Nigeria. Indian J Clin Biochem 2007;22:74-8.

10. Vigersky RA, Filmore-Nassar A, Glass AR. Thyrotropin Suppression by Metformin. J Clin Endocrinol Metabolism 2006;91:225-7.

11. Cappelli C, Rotondi M, Pirola. Thyrotropin Level in Diabetic Patients on Metformin Treatment. Eur $\mathrm{J}$ Endocrinol 2012;167:261-5.

12. Dias CM, Nogueira P, Rosa AN, et al. Total Cholesterol and High Density Cholesterol in Patients with Insulin Dependent Diabetes Mellitus. Acta Medica 1995;8:619-28.

13. Ravishankar SN, Champakamalini, Venkatesh, Mohsin. A Prospective Study of Thyroid Dysfunction in Patients with Type 2 Diabetes in General Population. imedPub Journal 2013; 5(1:2): 1-9.

14. Becker DV, Bigos ST, Gaitan E, et al. Optimal use of Blood Tests for Assessment of Thyroid Function. JAMA 1993;3:26973.

15. Demer LM, Spencer CA. Laboratory Medicine Practice. Laboratory Support for the Diagnoses and Monitoring of Thyroid Disease. Clin Endocrinol 2003;58:138-40.

16. Baloch Z, Carayon P, Conte-Davolx B, et al. Laboratory Medicine Practice Guidelines. Laboratory Support for the
Diagnosis and Monitoring of Thyroid Disease. Thyroid 2003; 13:3-126.

17. STATA statistical package version 13 (Stata Corp., College Station, Texas USA), 2016.

18. Haruna R, Attah YE, Purity NO. Understanding and Overcoming the Challenges of Youth Unemployment in Nigeria. Review of Public Administration and Management 2014;3:206-14.

19. Smithson MJ. Screening for thyroid dysfunction in a community population of diabetic patients. Diabet Med 1998;15:14850 .

20. Gursoy NT, Tuncel E. The relationship between the glycemic control and the hypothalamus-pituitary-thyroid axis in diabetic patients. Turkish Journal of Endocrinology and Metabolism 1999;4:163-8.

21. Radaideh ARM, Nusier MK, Amari FL. Thyroid Dysfunction in Patients with Type 2 Diabetes Mellitus in Jordan. Saudi Med J 2004;25:1046-50.

22. Akbar DH, Ahmed MM, Al-Mughales J. Thyroid Dysfunction and Thyroid Autoimmunity in Saudi Type 2 Diabetics. Acta Diabetologica 2006;43:14-8.

23. Perros P, McCrimmon RJ, Shaw G, Frier BM. Frequency of Thyroid Dysfunction in Diabetic Patients: Value of Annual Screening. Diabetic Med 1995;12:622-7.

24. Roche Diagnostics Ltd. Electro-chemiluminescence immunoassay (ECLIA) for the in vitro quantitative determination of free triodothyronine in human serum and plasma. T3 FactSheet. CH-6343 Rotkreuz, Switzerland. c2011 Roche.

25. Roche Diagnostics Ltd. Electro-chemiluminescence immunoassay (ECLIA) for the in vitro quantitative determination of free thyroxine in human serum and plasma. T4 FactSheet. CH-6343 Rotkreuz, Switzerland. c2011 Roche.

26. Elecsys TSH-Roche Diagnostics. Electro-chemiluminescence immunoassay (ECLIA) for the in vitro quantitative determination of thyrotropin in human serum and plasma. TSH FactSheet. CH-6343 Rotkreuz, Switzerland. c2011 Roche.

27. Chen HS, Wu TE, Jap TS. Subclinical Hypothyroidism is a Risk Factor for Nephropathy and Cardiovascular Disease in Type 2 Diabetic Patients. Diabetic Med 2007;24:1336-44.

28. Yang JK, Liu W, Shi J, Li YB. An Association between Subclinical Hypothyroidism and Sight-Threatening Diabetic Retinopathy in Type 2 Diabetic Patients. Diabetic Care 2010;33:1018-20.

29. Swamy RM, Kumar N, Srinivas K, et al. Evaluation of hypothyroidism as a complication of type 2 diabetes mellitus. Biomed Res 2012;23:170-2.

30. Murray RK, Granner DK, Mayes PA, Rodwell VW. Harper's Biochemistry. 25th edition. London: Prentice-Hall International Inc; 2000, p. 533-8.

31. Hage M, Zantout MS, Azar ST. Thyroid Disorders and Diabetes Mellitus. J Thyroid Res 2011;2011:439463. 